\title{
Cooperative Voltage Control Method by Residential PV Systems in Distribution System
}

\author{
Shoji Kawasaki and Masaaki Koyama \\ Department of Electronics and Bioinformatics, Meiji University, Kanagawa 214-8571, Japan
}

Received: December 16, 2015 / Accepted: January 05, 2016 / Published: February 29, 2016.

\begin{abstract}
Recently, the number of system interconnection of the renewable energy such as PV (photovoltaic) generation system and wind power generation system has been increasing drastically. A distribution system with the distributed generation must be operated keeping reliability of power supply and power quality. When high-capacity PV systems are interconnected to the distribution system, the system voltages may be deviated from the upper limit or lower limit of proper voltage in the distribution system. In this study, the authors propose a cooperative voltage control method of the distribution system by the power factor control of plural residential PV systems. In order to verify the validity of the proposal method, the numerical calculations are carried out by using an analytical model of distribution system which interconnected residential PV systems.
\end{abstract}

Key words: Photovoltaic generation, power quality, voltage control, distribution system, power factor.

\section{Introduction}

In recent years, the number of DG (distributed generation) using renewable energy sources such as $\mathrm{PV}$ system and wind power generation system that interconnects to a distribution system has been increasing to reduce the influence on the environment in Japan. When PV systems are interconnected to a distribution network, the line voltages may be deviated from the upper limit or lower limit of proper voltage in the distribution network. In the distribution system, it is necessary to keep reliability of power and power quality. PV systems provide the function to control the power factor in order to maintain the voltage of interconnecting point of PV systems in the distribution network. However, if the voltages of interconnecting point of PV system in the distribution network are controlled within the acceptable range, the voltage where PV system is not interconnected may be deviated from the upper limit or lower limit of

Corresponding author: Shoji Kawasaki, senior assistant professor, research fields: optimization control of distribution network and electric power quality improvement of distribution network. proper voltage. So far, the various studies on the voltage control method in the distribution network interconnecting DG have been studied [1-7].

In this paper, the authors propose a cooperative voltage control method of the distribution system by the power factor control of plural residential PV systems. As a result, all the point voltages of distribution system are controllable within the proper limits. In order to verify the validity of the proposal method, the numerical calculations are carried out by using an analytical model of distribution system which interconnected PV systems.

\section{Cooperative Voltage Control Method by PV Systems}

In general, only the voltage at interconnection point of PV system is monitored. And the output of PV is controlled or the power factor of interconnection of $\mathrm{PV}$ is controlled so as to fall the point voltage within the proper range, when the point voltage deviates from the voltage limit of distribution network. In the proposed method, the voltages of all interconnecting points of PV systems and the voltages of all points 
with IT (information technology) switch in the distribution network are monitored and controlled within the proper range by cooperated power factor control of plural PV systems when the point voltage of some point in the distribution network deviates from the voltage limit. Specifically, the dead band for power factor control by PV systems are set, and when a point voltage deviate from the upper limit or lower limit of dead band, the power factor of all PV systems which are interconnected to the deviated phase raise or down. And then, if the point voltage falls within the proper range, the power factor of PV systems is improved little by little. In addition, the power factor of PV systems is controlled within the limits of 0.85-1.00 set up by Japan's regulation of interconnection of DGs [8]. The control formulas and constraints required for the proposal method are shown in Eqs. (1)-(4), respectively.

$$
\begin{gathered}
V_{k L} \leq V_{k a b t} \leq V_{k H} \\
\text { If } V_{P V i t}>V_{P V_{-} H}^{*} \\
\cos \theta_{P V i a b t}^{*}=\cos \theta_{P V i a b t}-\Delta P F \\
\text { If } V_{P V i t}<V_{P V_{-} L}^{*} \\
\cos \theta_{P V i a b t}^{*}=\cos \theta_{\text {PViabt }}+\Delta P F \\
0.85 \leq \cos \theta_{\text {PViabt }} \leq 1.00
\end{gathered}
$$

where, $V_{k a b t}(\mathrm{~V})$ is line voltage of phase $a-b(=U-V$, $V-W, W-U)$ of point $k$ at time $t(\mathrm{~s}), V_{k H}, V_{k L}(\mathrm{~V})$ are upper limit and lower limit of the proper range of $V_{k a b t}$, $V_{P V i t}(\mathrm{~V})$ is point voltage of $\mathrm{PV}$ system $i(=1 \sim N)$ at time $t(\mathrm{~s}), V_{P V_{-} H}^{*}, V_{P V_{-} L}^{*}(\mathrm{~V})$ are upper limit and lower limit of the dead band for power factor control by PV systems, $\cos \theta_{\text {PViabt }}^{*}$ is command of power factor of PV system $i(=1 \sim N), \cos \theta_{\text {PViabt }}$ is power factor of $\mathrm{PV}$ system $i(=1 \sim N), \triangle P F$ is minute control variate of power factor.

The control procedure of proposed method and the conventional method for comparison are explained below.

(Proposed method)

[Procedure 1] Monitor the secondary voltages of pole transformer which residential PV systems are interconnected.

[Procedure 2] If a point voltage deviates from the upper limit of dead band, the power factor of all PV systems which are interconnected to the deviated phase are reduced by $\triangle P F$, and go to Procedure 1. If a point voltage deviates from the lower limit of dead band, the power factor of all PV systems which are interconnected to the deviated phase are raised by $\triangle P F$, and go to Procedure 1 . If the all point voltages fall within the dead band, go to Procedure 3.

[Procedure 3] If the power factor of PV systems is less than 1.00, the power factor of PV systems is raised by $\triangle P F$, and goes to the Procedure 1 .

(Conventional method)

Each PV system monitors the voltage at the interconnection point of PV system independently. And if the voltage is deviated from the proper range, the power factor of only the interconnection point will be controlled by the PV system.

The conceptual diagram of proposal method is shown in Fig. 1. In this system, the transmission of the information and the control signal are used by communication line. Therefore, if the distribution system becomes large, the delay time of control will occur. In this paper, the authors set the delay time of control $(\Delta t)$ and verify the relation between delay time of control and control effect. 


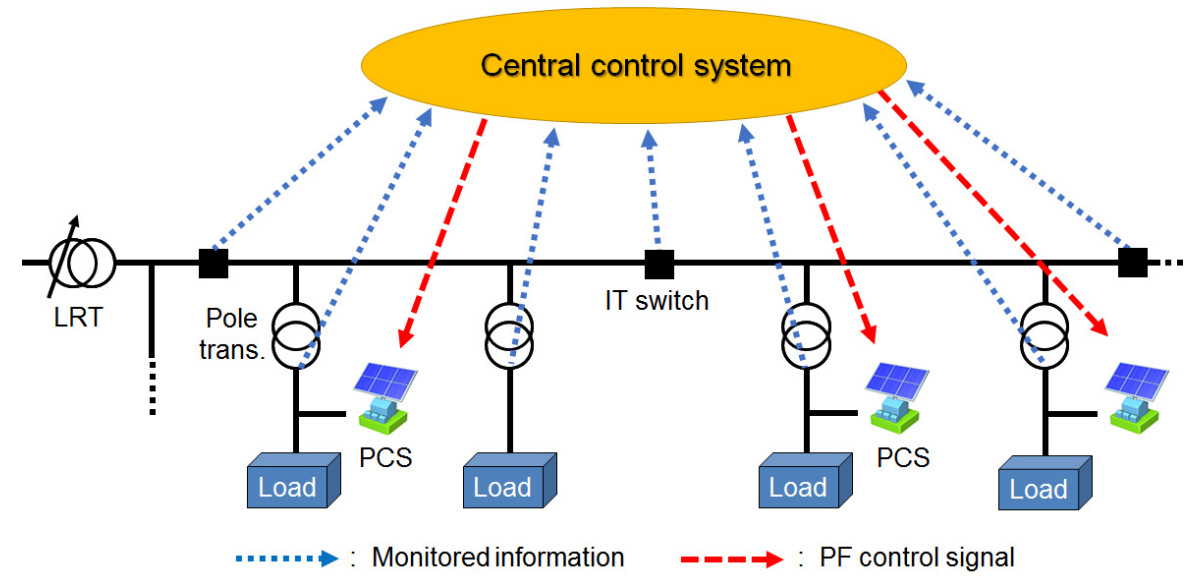

Fig. 1 Conceptual diagram of proposed method.

\section{Numerical Calcuation Example}

In order to verify the validity of the proposal method, the numerical calculations are carried out by using an analytical model of distribution system which interconnected plural residential PV systems.

\subsection{Analytical Model of Distribution System and Simulation Condition}

The distribution diagram which consists of two feeders used for analyses is shown in Fig. 2. The feeder 1 consists of 8 nodes. The total line length is $3.0 \mathrm{~km}$. And 8 loads, 6 switches, 8 pole transformers and 9 PV systems are connected to the feeder 1 . The feeder 2 consists of 6 nodes. The total line length is $2.0 \mathrm{~km}$. And 6 loads, 5 switches, 6 pole transformers and 9 PV systems are connected to the feeder 2 . The used loads are the residential load and commercial load based on actual measurement data. $100 \mathrm{~V}$ or $200 \mathrm{~V}$ loads are connected to the low-voltage side of distribution system. The total load capacity of feeder 1 and feeder 2 is $2 \mathrm{MW}$ and 1.6 MW, respectively. The total output of PVs is $50 \%$ of the total load capacity. The sending voltage of distribution system is $6,900 \mathrm{~V}$, and this model assumes the secondary voltage of pole transformer deviates from the voltage limit of distribution network $\left(V_{\text {Uuper }}=1.07\right.$ p.u. $(107 \mathrm{~V}), V_{\text {Lower }}=0.95$ p.u. $\left.(95 \mathrm{~V})\right)$.

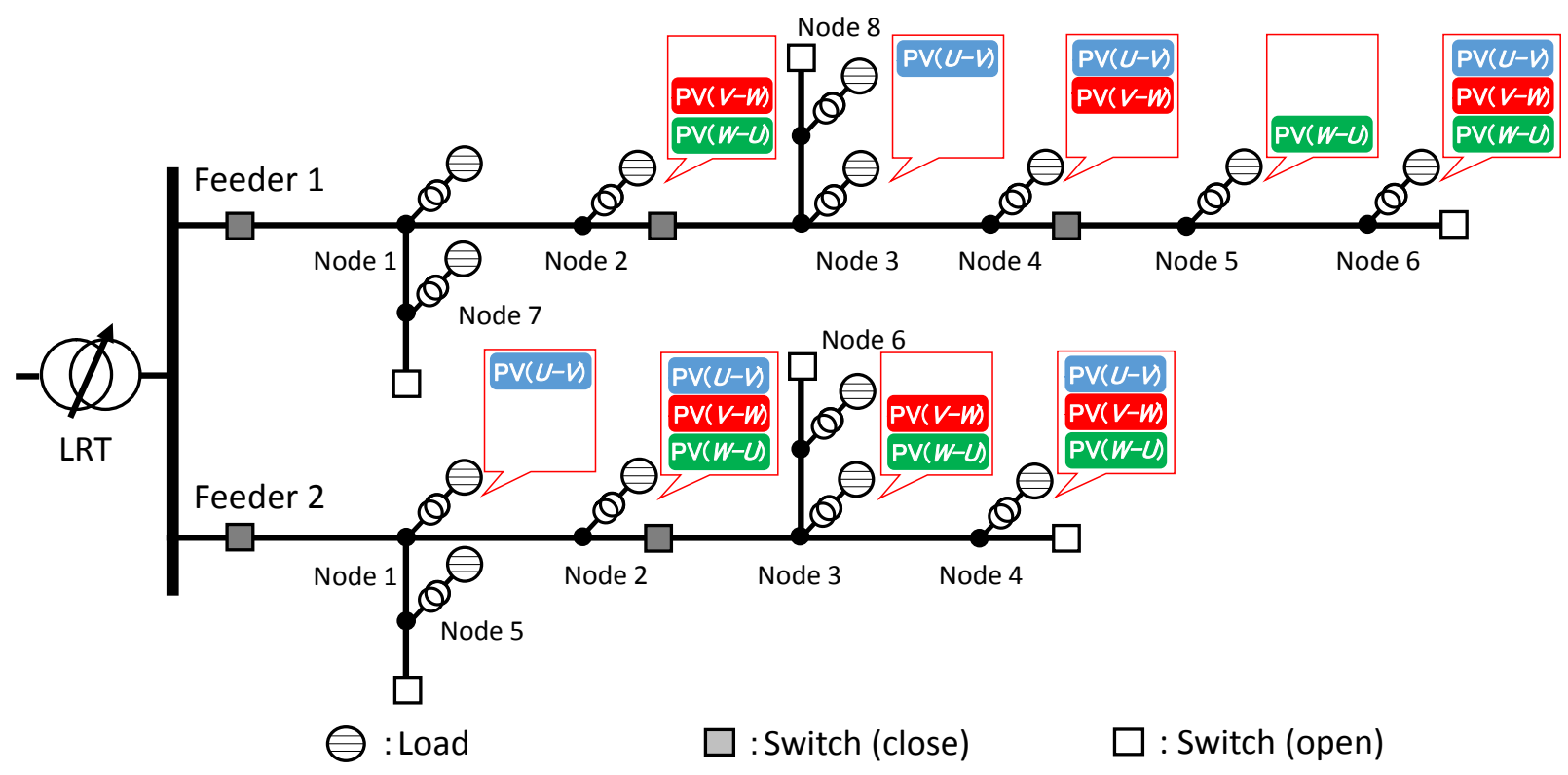

Fig. 2 Analytical model of distribution system. 


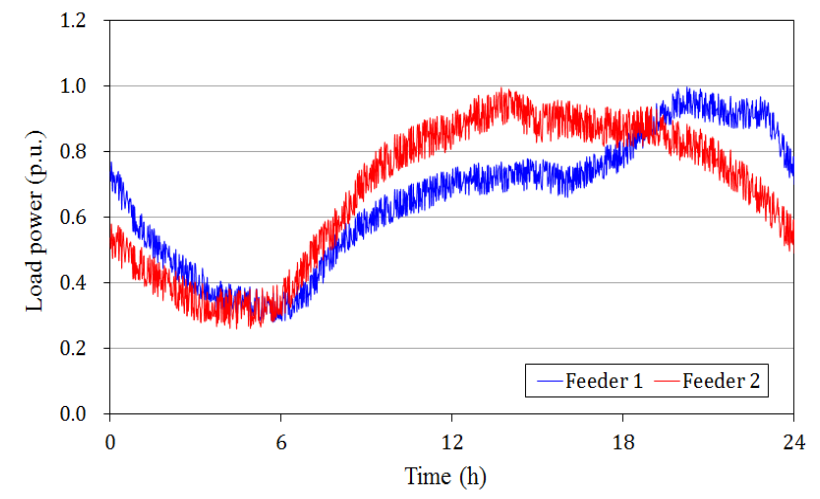

Fig. 3 Load curve.

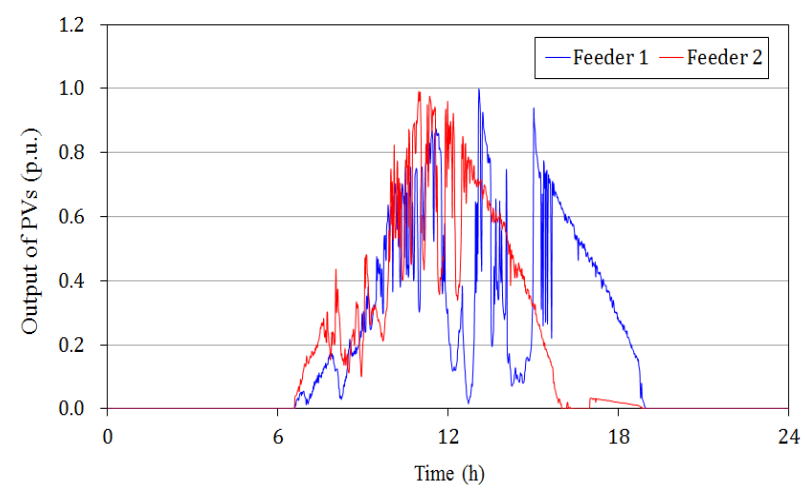

Fig. 4 Output of interconnected PVs.

The LRT (load ratio control transformer) in the analytical model is controlled LDC (line drop compensator) method. A number of taps of LRT is 9 . The used PV systems are single phase and assume a residential PV system. In this study, these PV systems are modeled as the current source. The dead band for power factor control by PV systems are set $V_{P V_{-} H}^{*}=$ 1.06 p.u. and $V_{P V_{L}}^{*}=0.96$ p.u..

The load curve used for the numerical calculation is shown in Fig. 3 and the output of interconnected PVs is shown in Fig. 4. In this study, the numerical calculations are carried out between 8:00 to $17: 00$ which PVs generate.

\subsection{Simulation Results}

The voltage control results at node 5 of feeder 1 by the conventional method and proposed method are shown in Fig. 5. As shown in Fig. 5, in conventional method, the voltage of interconnecting point of PV (phase WU) is controlled within the acceptable range.

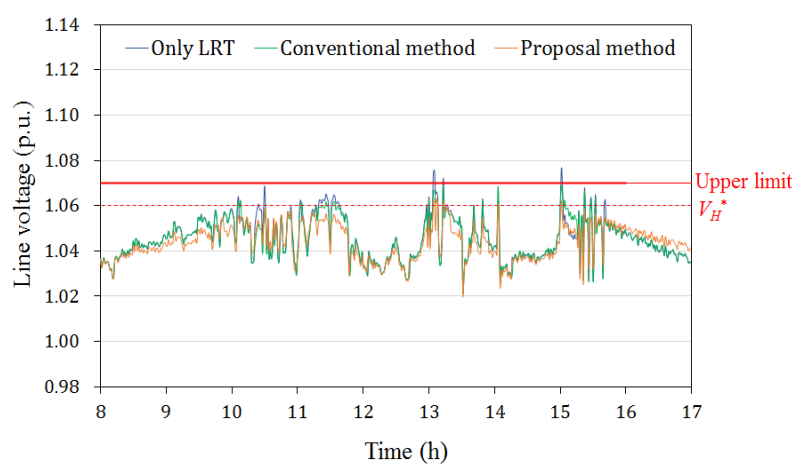

(a) Phase UV

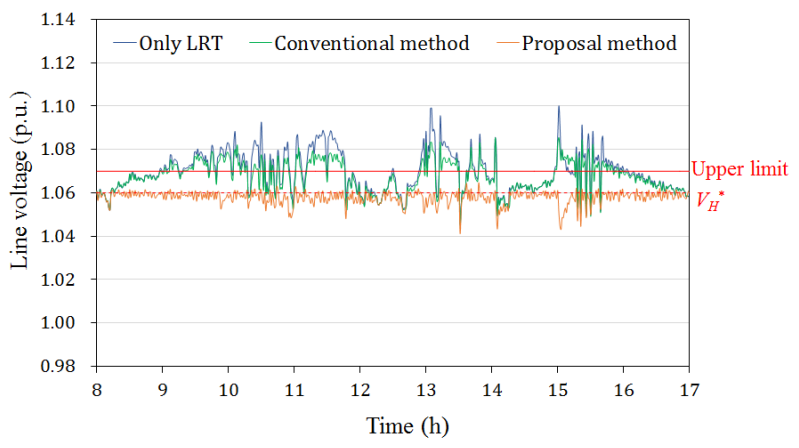

(b) Phase VW

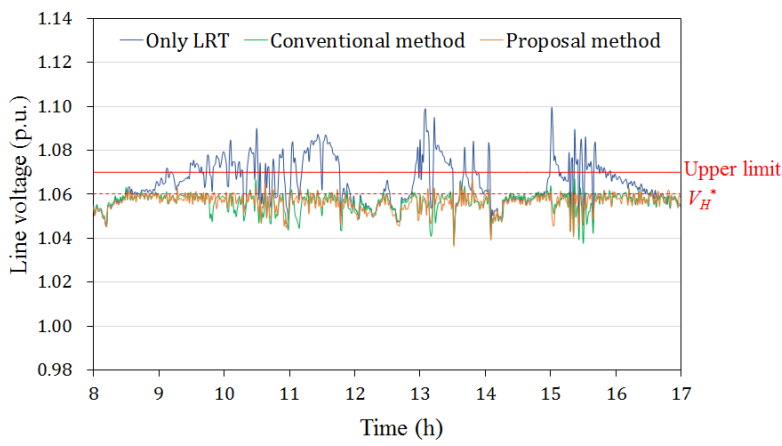

(c) Phase WU

Fig. 5 Comparison of suppressive effect on voltage deviation (feeder 1, node 5).

However, the voltage of unconnecting point of PV (phase VW) is not controlled within the acceptable range. On the other hand, in proposal method, the voltage of interconnecting point of $\mathrm{PV}$ is controlled within the acceptable range, and the voltage of unconnecting point of $\mathrm{PV}$ is also controlled within the acceptable range. Fig. 6 shows the simulation results of voltage control by the proposed method at the all nodes of feeder 1 and feeder 2. As shown in Fig. 6, the line voltages at all nodes are controlled within the acceptable range by the proposed method. 


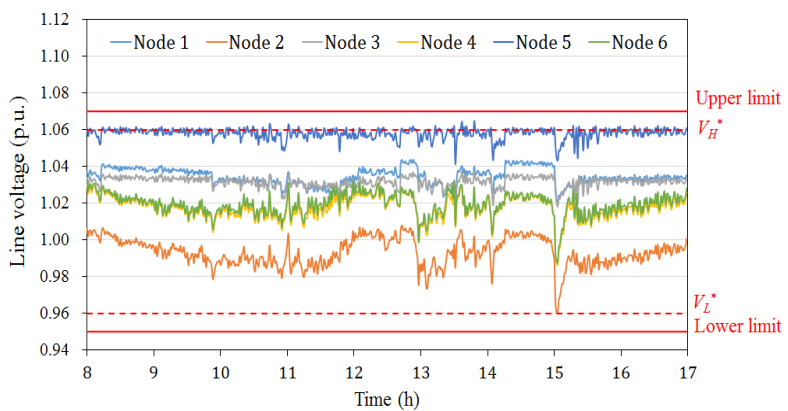

(a) Feeder 1

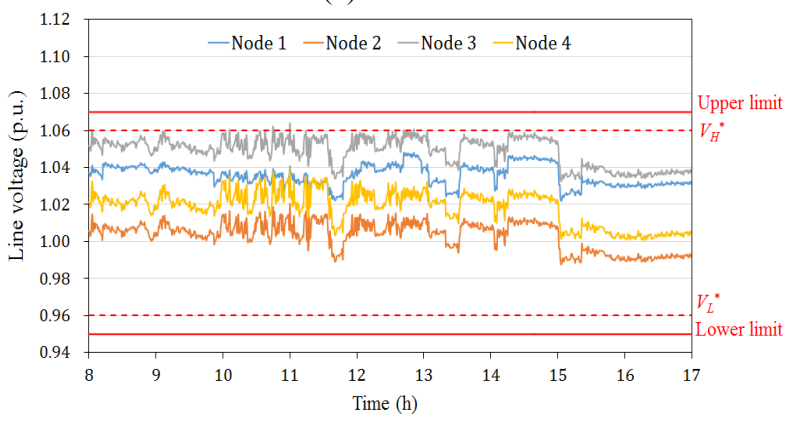

(b) Feeder 2

Fig. 6 Suppressive effect on voltage deviation by proposal method (phase VW).

Fig. 7 shows an improvement effect of maximum of voltage imbalance factor by each control method. In Fig. 7, the voltage imbalance factor are decreased by the proposal method in both of feeder 1 and feeder 2 . Fig. 8 shows the rate of active power output of PV systems. As shown in Fig. 8, the rate of active power output by the proposal method is lower than it by the conventional method especially in feeder 1 . This is because PV systems which the voltage of interconnecting point has not deviated are also controlling the system voltages in cooperation. Nonetheless, the rate of active power output of each PV systems by the proposal method is above $89 \%$. The proposal method optimizes the active power output of PV systems for control the system voltages.

Fig. 9 shows the transition of power factor of PV systems by the proposal method. In Fig. 9a, the power factor of all PV systems are quickly controlled to a steep variation of system voltages. In Fig. 9b, the power factor of PV systems interconnected to phase UV are not varying, because the voltages of phase UV are fall within the dead band. The power factor of PV

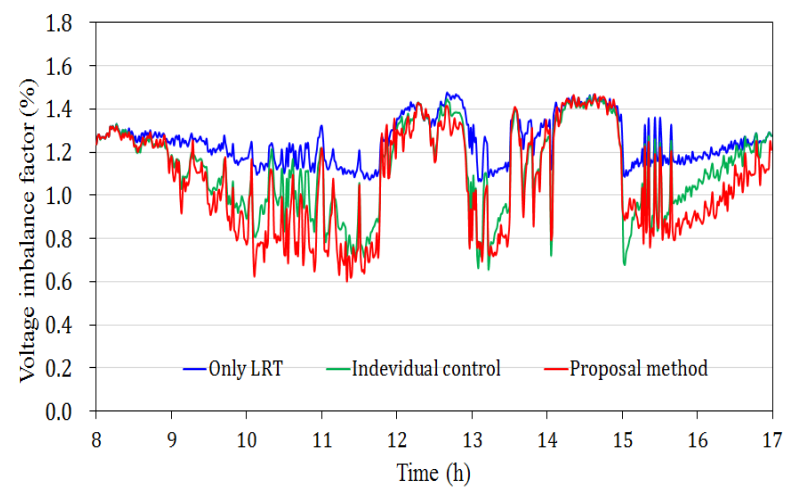

(a) Feeder 1

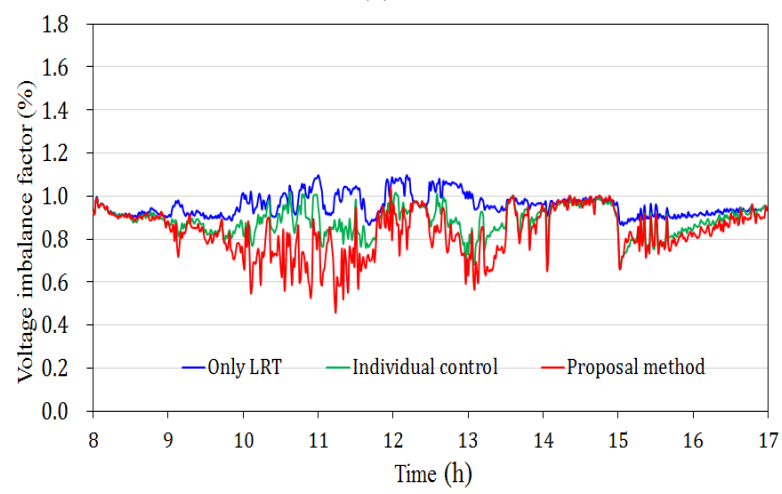

(b) Feeder 2

Fig. 7 Improvement effect of maximum of voltage imbalance factor.

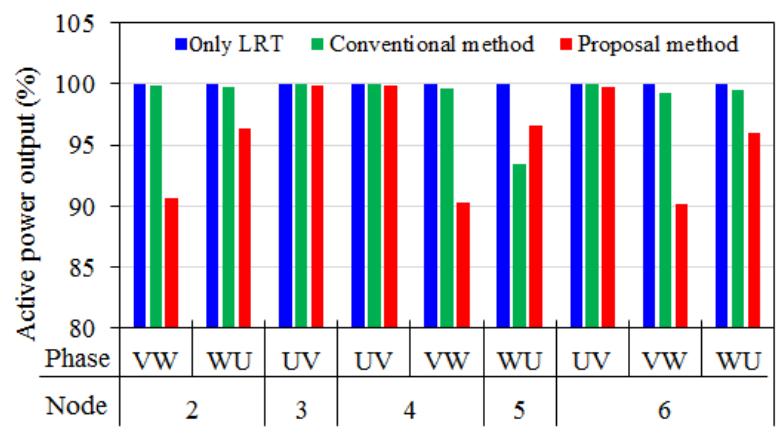

(a) Feeder 1

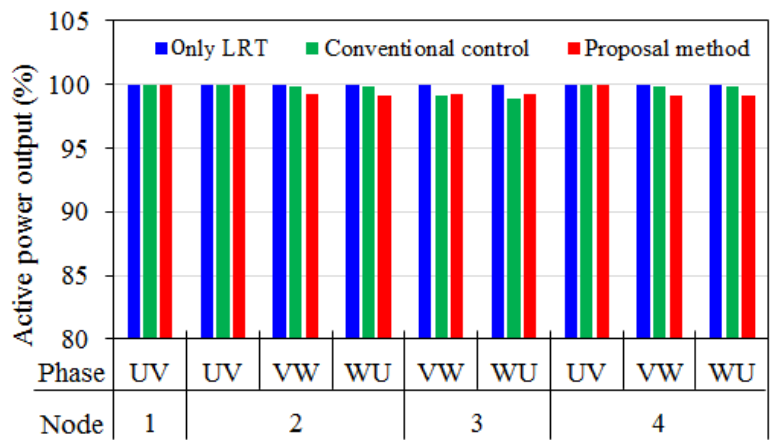

(b) Feeder 2

Fig. 8 Rate of active power output of PV systems. 


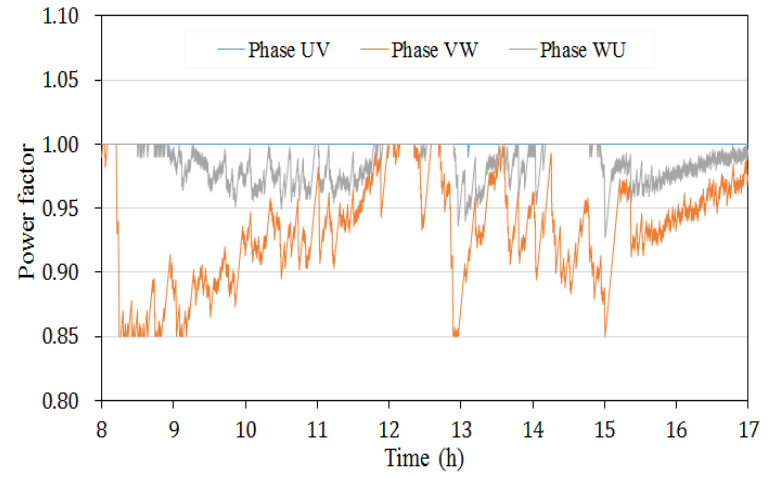

(a) Feeder 1

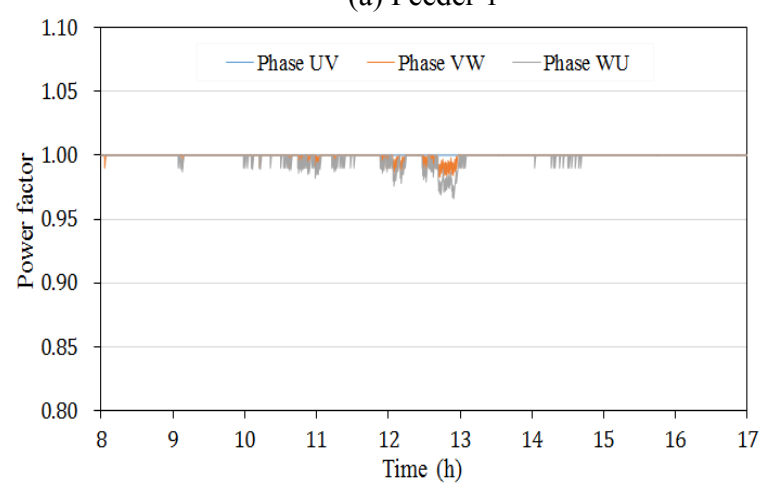

(b) Feeder 2

Fig. 9 Transition of power factor of PV systems by proposal method.

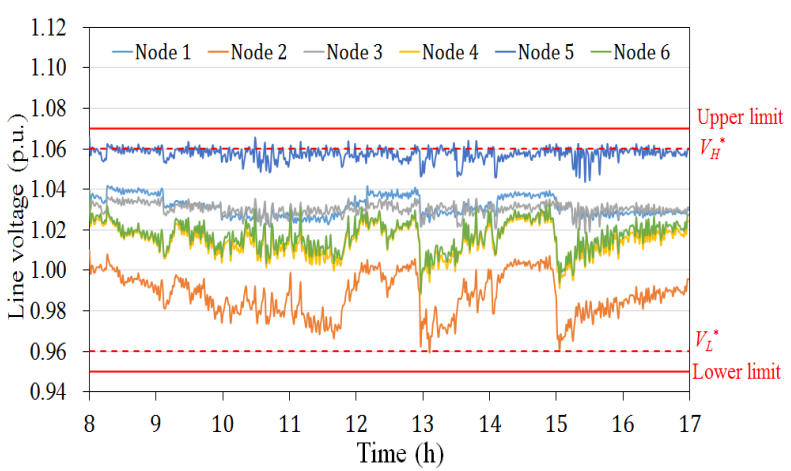

(a) $\Delta t=5 \mathrm{~s}$

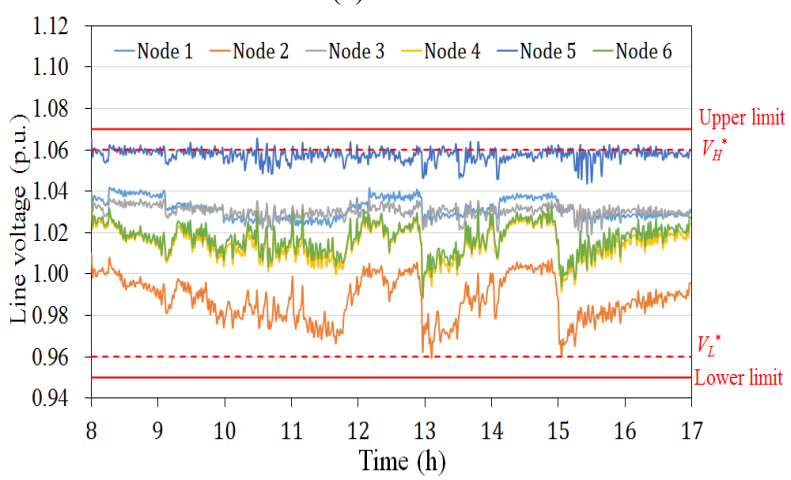

(b) $\Delta t=10 \mathrm{~s}$

Fig. 10 Voltage control effect in consideration of delay time of control.
Table 1 Deviation time from voltage limit.

\begin{tabular}{lllll}
\hline Case & No control & $\Delta t=0 \mathrm{~s}$ & $\Delta t=5 \mathrm{~s}$ & $\Delta t=10 \mathrm{~s}$ \\
\hline Time (s) & 3,405 & 13 & 60 & 123 \\
\hline
\end{tabular}

systems interconnected to phases VW and WU are controlled at high speed like it of feeder 1. Finally, the influence of the delay time $(\Delta t=0,5,10 \mathrm{~s})$ is verified. Fig. 10 shows voltage control effect in consideration of delay time of control and Fig. 6a shows the case $\Delta t$ $=0$ in comparison. Table 1 shows deviation time from voltage limit. As shown in Fig. 10 and Table 1, in case $\Delta t=10 \mathrm{~s}$, the deviation time from voltage limit is only $123 \mathrm{~s}$ out of the numerical calculations time 8:00-17:00. Even though the delay time occur, the line voltages at almost all nodes are controlled within the acceptable range by the proposed method.

\section{Conclusions}

In this paper, the authors proposed the cooperative voltage control method of the distribution system by the power factor control of plural residential PV systems. Specifically, the dead band for power factor control by PV systems were set, and when a point voltage deviates from the upper limit or lower limit of dead band, the power factor of all PV systems which are interconnected to the deviated phase raise or down. In order to verify the validity of the proposal method, the numerical calculations were carried out by using an analytical model of distribution system which interconnected residential PV systems. As a result of numerical calculations, the voltages of not interconnecting point of PV were not controlled within the acceptable range by the conventional method. On the other hand, the voltages of not interconnecting point of PV were controlled within the acceptable range by the proposal method. Moreover, the voltage imbalance in the distribution system was improved by the proposal method. Finally, the influence of the delay time of control was verified. Even though the delay time of control occurred, the line voltages were controlled within the acceptable range by the proposed method. 
The future work is to improve cooperative voltage control method corresponding to various configurations of distribution network and to carry out the numerical calculations in consideration of a control delay by the central control. Moreover, it is necessary to verify the usefulness of the proposed method by the experiment using a laboratory equipment of scaled-down distribution system with PV systems.

\section{References}

[1] Iioka, D., Sakakibara, K., Yokomizu, Y., Matsumura, T., and Izuhara, N. 2006. "Distribution Voltage Rise at Dense Photovoltaic Power Generation Area and Its Suppression by SVC.” IEEJ Trans. PE 126-B (2): 153-8.

[2] Oshiro, M., Senjyu, T., Yona, A., Urasaki, N., and Funabashi, T. 2010. "Reactive Power Control Method for Reducing Voltage Fluctuation in Large-Scale Photovoltaic Systems." IEEJ Trans. PE 130 (11): 972-80.

[3] Uchiyama, N., Miyata, H., Ito, T., and Konishi, H. 2010. "Reactive Power Control Method for Reducing Voltage
Fluctuation in Large-Scale Photovoltaic Systems." IEEJ Trans. PE 130 (3): 297-304.

[4] Ishimaru, M., Tamachi, H., and Komami, S. 2011. "A Study on Voltage Rise Control Effect by Leading Power Factor Operation of PVs Considering Tap Change of Distribution Transformer." IEEJ Trans. PE 131 (5): 429-36.

[5] Kawasaki, S., Kanemoto, N., Taoka, H., and Matuski, J. 2012. "Cooperative Voltage Control Method by Power Factor Control of PV Systems and LRT." IEEJ Trans. PE 132 (4): 309-16.

[6] Viawan, F. A., and Karlsson, D. 2008. "Voltage and Reactive Power Control in Systems with Synchronous Machine-Based Distributes Generation." IEEE Trans. Power Delivery 23 (2): 1079-87.

[7] Carvalho, P. M. S., Correia, P. F., and Ferreira, L. A. F. M. 2008. "Distributed Reactive Power Generation Control for Voltage Rise Mitigation in Distribution Networks.” IEEE Trans. Power Syst. 23 (2): 766-72.

[8] Interconnection of Power System Expert Committee of Japan Electric Association. 2012. "Interconnection of Power System Regulation JEAC 9701-2012.” Interconnection of Power System Expert Committee of Japan Electric Association. 